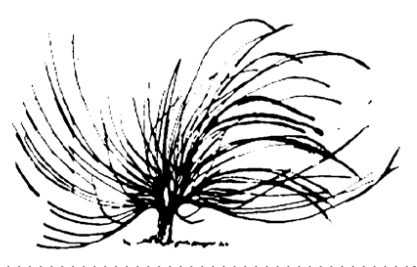

\title{
Instrumento de medición de la competencia de comunicación a través de estrategias metacognitivas en estudiantado universitario
}

\author{
Augusto A. Vargas Schüler ${ }^{1}$ \\ Universidad Técnica Federico Santa María \\ Chile \\ augusto.vargas@usm.cl \\ María B. Aguilar Bustos ${ }^{2}$ \\ Universidad Técnica Federico Santa María \\ Chile \\ maria.aguilar@usm.cl
}

\begin{abstract}
Resumen
El presente artículo reporta los resultados de una primera aproximación al diseño y validación de un instrumento que permita medir algunos aspectos que debiera desarrollar el estudiantado universitario perteneciente a una carrera técnica o de ingeniería y al menos desde su segundo año, en cuanto a la competencia comunicativa desde la mirada metacognitiva. El instrumento diseñado y validado corresponde a un cuestionario que consta de 26 preguntas aplicado como piloto en un grupo compuesto de 100 estudiantes. Las preguntas fueron agrupadas en cuatro dimensiones definidas como conocimiento, planificación, monitoreo/seguimiento y evaluación. El resultado mediante análisis factorial muestra que estos 4 componentes explican cerca del $47 \%$ de la varianza global,
\end{abstract}

Recibido: 20 de agosto de 2020. Aprobado: 1 de junio de 2021

http://dx.doi.org/10.15359/rep.16-2.13

1 Profesor Titular, Departamento Diseño y Manufactura, Universidad Técnica Federico Santa María - Chile. https://orcid.org/0000-0001-6023-1706

2 Profesora Adjunta, Departamento de Ciencias, Universidad Técnica Federico Santa María Chile. https://orcid.org/0000-0002-4598-4301 
donde el conocimiento es la más relevante en ese aspecto. El parámetro alfa de Cronbach es adecuado para el instrumento, y presenta un valor numérico 0.876 . La prueba KMO de adecuación de muestreo presenta un valor numérico de 0.787 , también adecuado para el instrumento.

Palabras clave: Comunicación, confiabilidad, instrumento, metacognición

\begin{abstract}
This paper reports the result of a first approach to the design and validation of an instrument which allows measuring some aspects that should be developed by a university student regarding communicative competence from a metacognitive perspective. The designed and validated instrument corresponds to a survey consisting of 26 questions piloted in a group of 100 students. The questions were grouped in four dimensions defined as knowledge, planining, monitoring/follow-up, and assesment. The result through factor analysis shows that these four components explain the $47 \%$ of the global variance, knowledge being the most relevant in that aspect. The Alpha Cronbach's parameter is suitable to the instrument, presenting a numerical value of 0.876 . The KMO sampling adequacy test has a numerical value of 0.787 , also suitable for the instrument.
\end{abstract}

Keywords: communication, instrument, metacognition, reliability

\title{
Introducción
}

$\mathrm{U}$

no de los desafíos más importantes en la educación radica en enseñar a aprender con el objetivo de lograr competencias. Lo anterior supone que es posible instruir en estudiantes las habilidades para desarrollar esa forma de pensamiento y lograr este fin. Una competencia, como concepto, se puede definir como el accionamiento e integración de conocimientos, habilidades y destrezas (Canales y Schmal, 2013) necesarias para saber qué, cómo, cuándo, por qué y para qué hacer en un determinado contexto laboral para desempeñarse exitosamente (Sáiz y Guijo 2010). Se pueden clasificar como específicas y genéricas, donde las primeras caracterizan a una profesión 
y la diferencian de otras, mientras que las genéricas son las habilidades necesarias para ejercer cualquiera de ellas (Arráez et al., 2008), a manera de ejemplo, el aprendizaje autónomo, el trabajo en equipo, la capacidad de resolución de problemas y las habilidades comunicativas (Verano-Tacoronte et al., 2016). Es esencial desarrollar estas últimas en el estudiantado universitario durante el tiempo en que esté en la universidad (Tovar, 2008a, 2008b), para poder aplicarlas en el manejo de las relaciones humanas, entendiendo distintos contextos, liderar grupos humanos, expresarse en público y fundamentar proyectos (Tejera y Cardoso, 2015).

La palabra comunicación deriva del latín communis que significa común, así, el comunicarse implica entrar en comunidad con alguien (Roca et al., 2005), al ser un proceso realizado en amplios aspectos de la vida, el cual permite intercambiar ideas, pensamientos, vivencias, emociones, sentimientos, donde los individuos responden y crean mensajes que les posibilita adaptarse a su entorno (De la Uz et al., 2009). El proceso comunicativo generalmente involucra cuatro elementos: emisor, receptor, canal y retroalimentación (Zanaton et al., 2012).

La comunicación verbal hace uso de palabras para emitir un mensaje, lo cual es esencial en los seres humanos, para desarrollarse a través de interacciones que las personas establecen entre sí (González et al., 2015). Sin embargo, las personas emiten mensajes como del estado del ánimo y carácter sin usar las palabras; estos últimos dos, ejemplos de la comunicación no verbal (Escobari, 2013). La comunicación no verbal se vale de expresiones faciales y movimientos del cuerpo, que acompañan a la emisión del mensaje (Rincón, 2011), que se pueden clasificarse en kinésica, proxémica, cronémica y paralenguaje: la primera y la última son las más importantes (Escobari, 2013). Así, parece ser que en todo momento el entorno nos ve comunicando, incluso al no efectuar actividad alguna.

La preparación en la comunicación interpersonal, la cual incluye la verbal (oral o escrita) y no verbal, resulta una prioridad en la formación del actual estudiantado universitario, para que, de este modo, pueda enfrentarse a la globalización multicultural (Jurado et al., 2017). En el ámbito educacional, las competencias se establecen no solo para lograr la coherencia de la enseñanza, sino también para alinear estas con el mundo laboral, al promoverse aquellas de carácter genérico como específico y potenciando aquellas necesarias para integrarse al 
mundo laboral (Verano-Tacoronte et al., 2016). Cuando se detecta que estudiantes que recién ingresan a la universidad evidencian alguna escasez en la competencia comunicación, estas se deben hacer cargo de esa dificultad, proponiendo acciones como, por ejemplo, cursos o talleres que permitan minimizar esa carencia (Rico y Níkleva, 2016).

La comunicación kinésica se relaciona con movimientos del rostro y posturas corporales que adquiere el cuerpo en alguna situación (Rincón, 2011). Sin lugar a dudas, este tipo de comunicación puede ser o no voluntaria y simbolizar un estado de ánimo, alguna predisposición, algún sentimiento o simplemente representar la manera en que como nos sentimos.

En general, el mayor porcentaje de la comunicación es realizada a través del lenguaje no verbal, seguida por la oral y por último mediante la comunicación escrita (Mehrabian 1981, según se cita en Rincón, 2011). Es de vital importancia detenerse en este punto, pues al parecer más de la mitad del volumen de información que transmitimos parece ser no verbal, lo que llevaría a pensar en el impacto que tendría conocer y potenciar las habilidades comunicativas en estudiantado egresado y titulado universitario.

La competencia comunicativa se puede definir como los conocimientos y las capacidades relacionadas con el uso de la lengua en distintos entornos (Marín, 2017), al respetar reglas, como, por ejemplo, el léxico y la gramática y otros aspectos en el medio en el cual se lleva a cabo la comunicación (Godoy, 2016). Asimismo, esta competencia está formada por un conjunto de subcompetencias interrelacionadas entre sí, a saber, competencia lingüística, competencia sociolingüística, competencia discursiva, competencia estratégica y competencia pragmática (Godoy, 2016).

Con el avance tecnológico, que se ha dado en el último tiempo (González et al., 2015), el inglés juega un papel importante, pues mucha documentación está escrita en este idioma, considerado de carácter universal (Troitiño y Hernández, 2015). En cuanto al proceso de enseñanza aprendizaje de esta lengua, se distinguen variadas acciones orientadas a mejorar el proceso de enseñanza aprendizaje, por ejemplo, a través de la promoción de la participación del estudiantado (Bonilla y Díaz, 2018), por medio de exposiciones, paneles de discusión, juegos de roles, entre otros (Troitiño y Hernández, 2015). El dominio de otro idioma, y en particular del inglés, requiere el conocimiento de un código lingüístico 
como competencia gramatical y entender la cultura que se necesita para establecer la comunicación (González et al., 2015) donde el estudiantado tiene que hacer algo más que proporcionar respuestas de una sola palabra o recitar frases aisladas en el idioma inglés (Ruiz et al., 2018). En Chile, el Ministerio de Educación enfatiza, a nivel escolar público, el desarrollo de la competencia comunicativa en inglés; sin embargo, las clases resaltan la repetición, traducción y el uso estructuras gramaticales (Yilorm, 2016), sobre otras que como la capacidad de entender y comunicarse en ese idioma.

Flavell (1976) define la cognición como el conocimiento propio respecto de sus propios procesos y productos cognitivos o cualquier otro asunto relacionado con ello. Se agrega, por otro lado, que la metacognición puede ser entendida como la supervisión, regulación y organización de procesos cognitivos en relación con la búsqueda de alguna meta $\mathrm{u}$ objetivo (Osses et al., 2010). Se considera como uno de los espacios de investigación que más han aportado a los nuevos pensamientos en torno al aprendizaje y la instrucción (Jaramillo y Osses 2012). Además, Schraw y Moshman (1995) señalan que la metacognición se compone tanto del conocimiento sobre la cognición como de la regulación de ella, donde el primero se refiere al conocimiento que posee cada individuo sobre su propio conocimiento y tiene como subprocesos los conocimientos declarativos (saber sobre), procedimental (saber cómo) y condicional (saber por qué y cuándo) (Huertas et al., 2014).

La regulación de los procesos cognitivos se lleva a cabo mediante tres aspectos: la planificación, en la cual se esbozan los actos a ejecutar y que finaliza con la elaboración de un plan de acción; la supervisión, en la cual se inspecciona el desarrollo de la actividad cognitiva, al comprobarse los avances y adoptarse medidas correctivas $\mathrm{y}$, finalmente, la evaluación, en la cual se evidencian las metas logradas y se señalan los problemas suscitados en el proceso (Jaramillo y Osses, 2012).

La metacognición puede ser medida mediante las rúbricas, autorreportes, cuestionarios y entrevistas (Bonilla y Díaz, 2018). Existen además otros instrumentos para medir la consciencia metacognitiva de los individuos como el Metacognitive Awareness Inventory (MAI) y el Metacognitive Awareness of Reading Strategies Inventory (MARSI). El Metacognitive Awareness Inventory (MAI) es un cuestionario en escala Likert creado en 1994 por Schraw y Dennison con la intención de identificar las habilidades metacognitivas mediante 52 reactivos distribuidos 
en las categorías conocimiento y regulación de la cognición (González et al., 2017). El MARSI o Metacognitive Awareness of Reading Strategies Inventory está conformado por 30 ítems y utiliza una escala Likert de 5 puntos creada por Mokhtari y Reichard (2002). En el instrumento, las estrategias de lectura están clasificadas en tres tipos: Estrategias de lectura globales (13 ítems), estrategias de resolución de problemas (8 ítems) y estrategias de apoyo (9 ítems) (Ramírez y Pereira, 2006).

El uso de estrategias metacognitivas para el aprendizaje se relaciona con la madurez intelectual de cada persona, pues la reflexión exige una autonomía de pensamiento (Tesouro, 2005). La consciencia y el desarrollo de los procesos cognitivos y metacognitivos en el estudiantado universitario puede influir positivamente en la adquisición y retención de conocimientos, resolución de problemas, en la comunicación oral, en la adquisición del lenguaje y varios tipos de autocontrol y autoinstrucción (Di Pardo, 2019; Palacios y Schinella, 2017).

En cuanto el aprendizaje de una segunda lengua, se puede mencionar un estudio realizado por Liyanage et al. (2012), quienes revelaron que el uso de estrategias metacognitivas influye positivamente en el aprendizaje de idiomas y que las estrategias metacognitivas utilizadas fueron la atención electiva, la planificación organizacional y el automanejo en tareas comunicativas de expresión oral y comprensión auditiva tanto fuera como dentro de la sala de clases (Bonilla y Díaz, 2018). 


\section{Metodología}

El objetivo de este trabajo fue diseñar y validar un instrumento que permita medir la competencia comunicativa en estudiantes universitarios, de nivel técnico o ingeniería, a través estrategias metacognitivas. El trabajo comprendió 5 etapas, las cuales se muestran en la Tabla 1.

\section{Tabla 1}

Etapas en el diseño del instrumento

\begin{tabular}{l}
\hline Etapa \\
\hline Etapa 1: Diseño del instrumento versión preliminar. \\
\hline Etapa 2: Sometimiento del instrumento versión preliminar a juicio de \\
expertos. \\
\hline Etapa 3: Aplicación del instrumento ajustado por los expertos en estudiantes como \\
prueba piloto. \\
\hline Etapa 4: Análisis estadístico de datos. Contempló análisis confiabilidad y validez de \\
constructo del instrumento. \\
Etapa 5: Construcción del instrumento final. \\
\hline Nota: Elaboración propia.
\end{tabular}

A continuación, se muestra el desarrollo de las etapas.

Etapa 1: Diseño del instrumento versión preliminar. En esta etapa, se diseñó y construyó un instrumento versión preliminar compuesto por 90 preguntas cerradas relacionadas con actitudes que pudieran presentar estudiantes hacia la competencia сотиnicación con un enfoque desde la estrategia metacognitiva. Las respuestas a las preguntas tuvieron 5 posibles respuestas excluyentes entre sí, en escala Likert y todas fueron redactadas en positivo. Las alternativas fueron: totalmente en desacuerdo, en desacuerdo, ni de acuerdo ni en desacuerdo, de acuerdo y totalmente de acuerdo. En esta etapa, no se consideraron a priori dimensiones del instrumento.

\section{Etapa 2: Sometimiento del instrumento preliminar a juicio} experto. En esta etapa, el Instrumento versión preliminar se sometió a un panel experto con el propósito de revisar todas las preguntas, corregir aquellas que requerían una mejor redacción 
y eliminar aquellas que no aportaban al objetivo del instrumento para generar el instrumento ajustado, con lo cual quedaron solo 52 preguntas. Paralelamente, se predefinieron 4 dimensiones: conocimiento, planificación, monitoreo/seguimiento y evaluación, las cuales agruparon a estas 52 preguntas.

Etapa 3: Aplicación del instrumento ajustado por el equipo experto en estudiantes como prueba piloto. El instrumento ajustado, compuesto de 52 preguntas, fue aplicado a 100 estudiantes pertenecientes a tres carreras pertenecientes a la Universidad Técnica Federico Santa María - Chile, y se salvaguardó la identidad de los sujetos encuestados.

Etapa 4: Análisis estadístico de datos. Los resultados de la encuesta, compuesta por 52 preguntas y aplicada como prueba piloto sobre 100 estudiantes, fueron sometidos a un análisis estadístico, de confiabilidad y validez de constructo del instrumento.

Confiabilidad del instrumento: Para ello, se calcularon los parámetros varianza, mínimo, máximo, media, alfa de Cronbach global para las 52 preguntas y alfa de Cronbach, si es eliminada una determinada pregunta. Las preguntas fueron configuradas como variables ordinales previo al análisis estadístico. Al considerar que el total de estudiantes contestó las 52 preguntas, se generó la estadística descriptiva, se calculó en primer lugar el alfa de Cronbach y en segundo lugar se realizó el análisis factorial respectivo. Para cada prueba estadística se utilizó un intervalo de confianza del $95 \%$, es decir $\alpha=5 \%$. El resultado del primer análisis se muestra en la Tabla 2.

Tabla 2

Análisis estadístico de fiabilidad para las 52 preguntas

\begin{tabular}{|l|l|}
\hline \multicolumn{2}{|l|}{ Estadísticas de fiabilidad } \\
\hline Alfa de Cronbach & $\mathrm{N}$ de elementos \\
\hline 0.914 & 52 \\
\hline
\end{tabular}

Nota: Elaboración propia. 
Un valor numérico del alfa de Cronbach por sobre 0.75 se considera suficiente para aceptar la fiabilidad del instrumento y en este caso el valor global es 0.914 , el cual lo supera con creces; se concluye que el instrumento en general puede considerarse fiable. Al analizar aquellas preguntas que aumentan en el valor numérico del alfa de Cronbach, si estas fueran eliminadas, los resultados se muestran en la Tabla 3.

\section{Tabla 3}

Alfa de Cronbach si el elemento es suprimido

\begin{tabular}{lccc}
\hline & $\begin{array}{c}\text { Media de escala si } \\
\text { el elemento se ha } \\
\text { suprimido }\end{array}$ & $\begin{array}{c}\text { Varianza de escala } \\
\text { si el elemento se } \\
\text { ha suprimido }\end{array}$ & $\begin{array}{c}\text { Alfa de Cronbach } \\
\text { si el elemento se } \\
\text { ha suprimido }\end{array}$ \\
\hline Pregunta 3 & 127.15 & 863.765 & 0.915 \\
\hline Pregunta 14 & 126.22 & 867.931 & 0.915 \\
\hline Pregunta 24 & 127.05 & 865.907 & 0.915 \\
\hline Pregunta 28 & 127.18 & 866.917 & 0.915 \\
\hline Pregunta 50 & 127.07 & 870.227 & 0.916 \\
\hline Pregunta 51 & 127.40 & 872.525 & 0.915 \\
\hline
\end{tabular}

Nota: Elaboración propia.

De los resultados mostrados en la Tabla 3, se desprende que se deben eliminar 6 de las 52 preguntas, con lo cual el instrumento se reduce solo a 46 preguntas, a las cuales se le aplicará el análisis factorial con la intención de determinar la validez del constructo.

Validez de constructo del instrumento: Se realizó el análisis factorial, mediante el método de componentes principales a través de la rotación Varimax, fijándose como 4 el número de factores a extraer. Así, se realizó la prueba Kaiser-Meyer-Olkin (KMO) y la prueba de esfericidad de Bartlett de adecuación de muestreo, se calculó la varianza total, la gráfica de sedimentación, el alfa de Cronbach y la matriz de cargas factoriales.

En la Tabla 4, se muestran los resultados correspondientes a la prueba KMO y esfericidad de Bartlett para las 46 preguntas. 


\section{Tabla 4}

Prueba KMO y Bartlett para las 46 preguntas

\begin{tabular}{|l|l|l|}
\hline \multicolumn{2}{|l|}{ Prueba de KMO y Bartlett } \\
\hline Medida Kaiser-Meyer-Olkin de adecuación de muestreo & 0.722 \\
\hline \multirow{2}{*}{$\begin{array}{l}\text { Prueba de esfericidad de } \\
\text { Bartlett }\end{array}$} & $\sim x^{2}$ & 2155.265 \\
\cline { 2 - 3 } & gl & 1035 \\
\cline { 2 - 3 } & Sig. & 0.000 \\
\hline
\end{tabular}

Nota: Elaboración propia.

En referencia a la Tabla 4, se observa que el valor numérico es 0.722 , con lo cual se entiende como validado el constructo del instrumento, aunque se hubiera esperado un valor numérico superior a 0.8. Paralelamente la significancia cumple con la condición menor a 0.05 para poder aplicar el análisis factorial, siendo en este caso 0 .

En la Tabla 5, se observa la varianza explicada por los 4 componentes elegidos en el análisis factorial. 


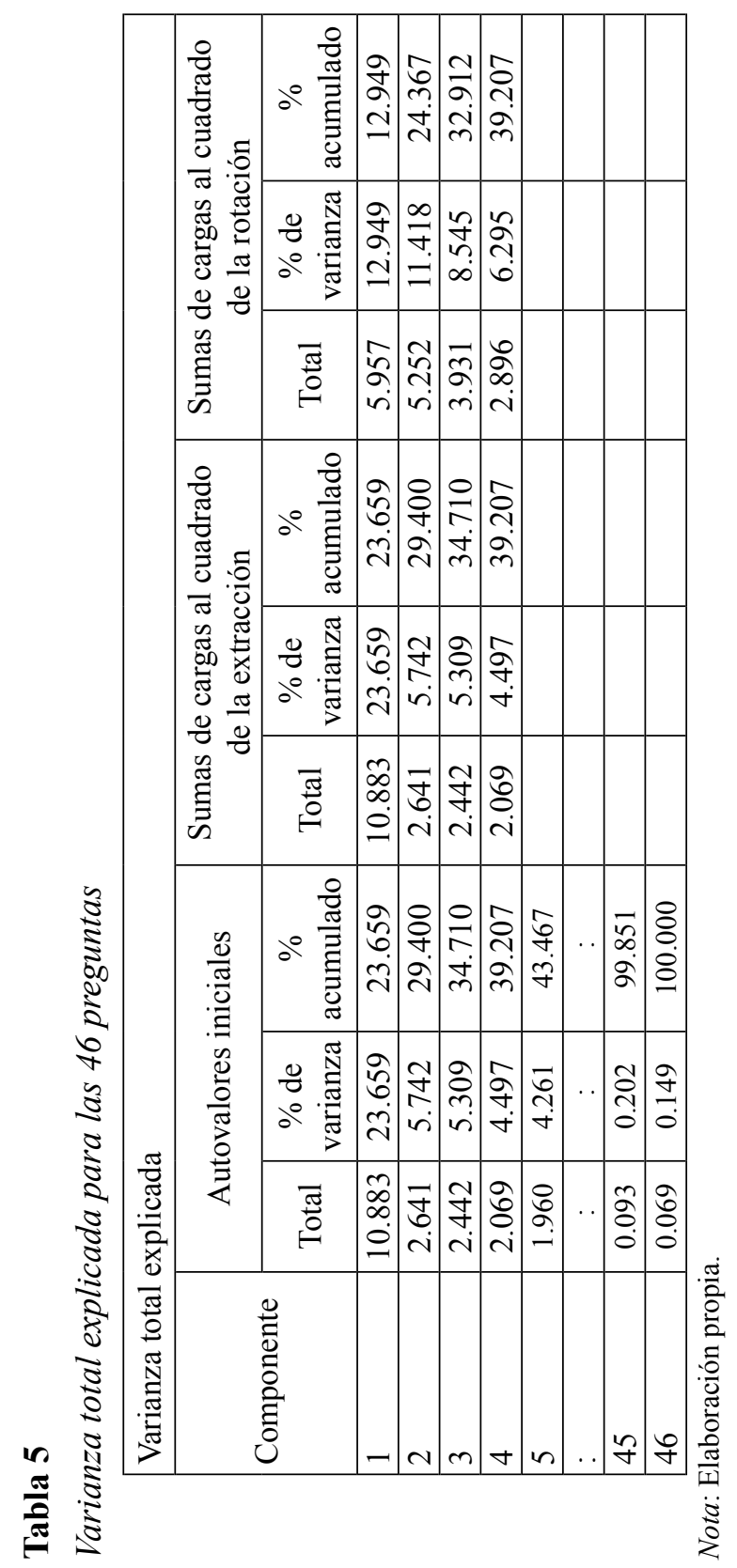


En la Tabla 5, se observa que el componente 1, componente 2, componente 3 y componente 4 explican el $23.66 \%, 5.74 \%, 5.31 \%$ y $4.5 \%$ de la varianza total respectivamente. Los 4 componentes explican solo un $39.21 \%$ de la varianza total, el cual puede considerarse bastante bajo. El gráfico de sedimentación se muestra en la Figura 1; se desprende que el componente 1 explica por si solo la varianza total, aunque con un valor numérico bastante pobre.

\section{Figura 1}

Gráfico de sedimentación para las 46 preguntas

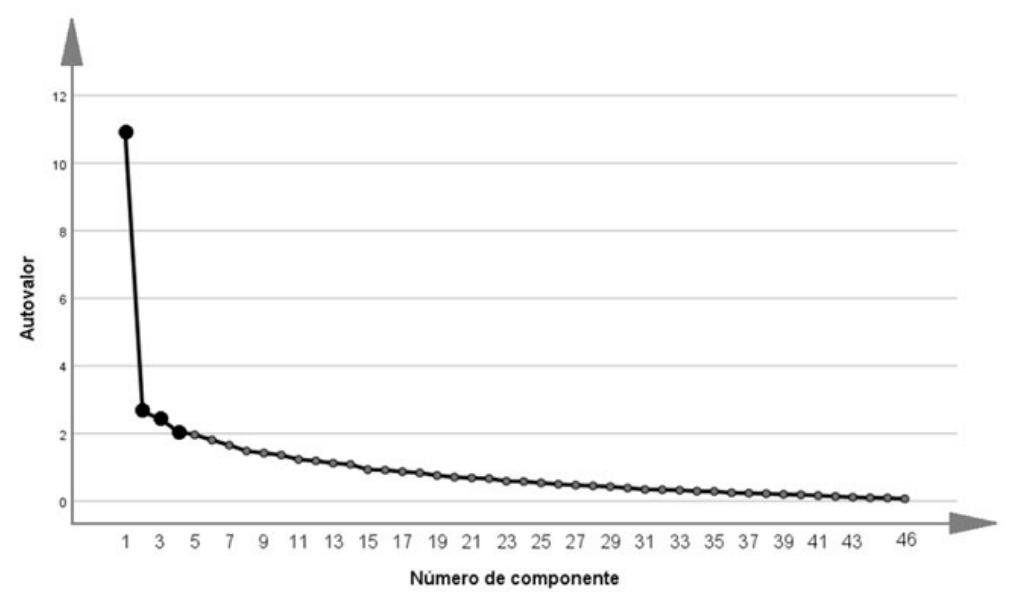

Nota: Elaboración propia.

En la Tabla 6, se muestra el Alfa de Cronbach para las 46 preguntas finales. Se observa que el valor creció respecto al resultado anterior con 52 preguntas, aumentando desde 0.914 a 0.922 , el cual se considera un valor numérico alto.

\section{Tabla 6}

Alfa de Cronbach para las 46 preguntas

\begin{tabular}{|l|l|}
\hline \multicolumn{2}{|c|}{ Estadísticas de fiabilidad } \\
\hline Alfa de Cronbach & $\mathrm{N}$ de elementos \\
\hline 0.922 & 46 \\
\hline
\end{tabular}

Nota: Elaboración propia. 
En la Tabla 7, se muestra la matriz de componentes rotados. Se observa que ciertas preguntas del constructo presentan cargas factoriales estadísticamente significativas, con valores mayores a 0.5 agrupadas, en su mayoría, en el primer componente; sin embargo, existen otras que presentan cargas inferiores a 0.5 , incluso algunas muy cercanas a 0 . De esa manera, se eliminarán las preguntas cuyas cargas factoriales son inferiores a 0.5 , las cuales se indican con un asterisco.

\section{Tabla 7}

Matriz de componentes rotados para las 46 preguntas

\begin{tabular}{|c|c|c|c|c|}
\hline \multicolumn{5}{|c|}{ Matriz de componentes rotados } \\
\hline & \multicolumn{4}{|c|}{ Componente } \\
\hline & 1 & 2 & 3 & 4 \\
\hline Pregunta $1 *$ & 0.125 & 0.461 & 0.296 & -0.007 \\
\hline Pregunta $2 *$ & 0.111 & 0.201 & 0.454 & 0.102 \\
\hline Pregunta $4 *$ & -0.109 & 0.431 & 0.270 & 0.037 \\
\hline Pregunta 5 & 0.106 & 0.631 & 0.226 & 0.075 \\
\hline Pregunta $6 *$ & 0.242 & 0.407 & 0.260 & -0.034 \\
\hline Pregunta $7 *$ & 0.347 & 0.135 & 0.499 & -0.058 \\
\hline Pregunta 8 & 0.114 & 0.132 & 0.636 & 0.084 \\
\hline Pregunta 9 & 0.033 & 0.200 & -0.019 & 0.567 \\
\hline Pregunta 10 & 0.123 & 0.581 & 0.293 & -0.097 \\
\hline Pregunta $11 *$ & 0.152 & 0.481 & -0.002 & 0.045 \\
\hline Pregunta 12 & 0.003 & 0.092 & 0.031 & 0.566 \\
\hline Pregunta 13 & 0.241 & 0.601 & 0.125 & 0.166 \\
\hline Pregunta $15 *$ & 0.263 & 0.258 & 0.496 & 0.062 \\
\hline Pregunta $16 *$ & 0.240 & 0.338 & 0.326 & 0.162 \\
\hline Pregunta $17 *$ & 0.351 & 0.197 & 0.430 & 0.225 \\
\hline Pregunta $18 *$ & 0.086 & 0.494 & 0.384 & -0.093 \\
\hline Pregunta $19 *$ & 0.418 & 0.376 & 0.473 & -0.063 \\
\hline Pregunta $20 *$ & -0.021 & 0.433 & 0.474 & 0.028 \\
\hline Pregunta $21 *$ & 0.432 & 0.122 & 0.298 & -0.210 \\
\hline Pregunta $22 *$ & 0.463 & -0.065 & 0.263 & -0.106 \\
\hline Pregunta 23 & 0.155 & 0.072 & 0.189 & 0.536 \\
\hline Pregunta $25 *$ & 0.043 & 0.498 & 0.146 & 0.125 \\
\hline Pregunta 26 & 0.599 & -0.001 & 0.111 & 0.124 \\
\hline Pregunta 27 & 0.329 & 0.188 & 0.532 & 0.258 \\
\hline
\end{tabular}




\begin{tabular}{lcccc}
\hline \multicolumn{5}{c}{ Matriz de componentes rotados } \\
\hline & \multicolumn{5}{c}{ Componente } \\
\hline & $\mathbf{1}$ & $\mathbf{2}$ & $\mathbf{3}$ & $\mathbf{4}$ \\
\hline Pregunta 29 & 0.061 & 0.514 & 0.280 & 0.082 \\
\hline Pregunta 30 & 0.549 & 0.114 & -0.085 & 0.275 \\
\hline Pregunta 31 & 0.341 & 0.654 & 0.199 & 0.086 \\
\hline Pregunta 32 & 0.126 & 0.560 & -0.098 & 0.161 \\
\hline Pregunta 33 & 0.019 & -0.106 & 0.097 & 0.627 \\
\hline Pregunta 34 & 0.344 & 0.140 & -0.179 & 0.279 \\
\hline Pregunta 35 * & 0.216 & -0.051 & 0.453 & 0.421 \\
\hline Pregunta 36 * & 0.497 & 0.266 & 0.274 & 0.259 \\
\hline Pregunta 37 & 0.520 & 0.240 & 0.354 & 0.089 \\
\hline Pregunta 38 * & 0.321 & 0.247 & -0.204 & 0.112 \\
\hline Pregunta 39 & 0.188 & 0.105 & -0.179 & 0.534 \\
\hline Pregunta 40 & -0.062 & 0.057 & 0.364 & 0.630 \\
\hline Pregunta 41 & 0.385 & 0.589 & -0.090 & 0.025 \\
\hline Pregunta 42 & 0.561 & 0.156 & 0.188 & 0.056 \\
\hline Pregunta 43 & 0.646 & 0.237 & 0.073 & 0.082 \\
\hline Pregunta 44 & 0.611 & 0.048 & 0.045 & 0.034 \\
\hline Pregunta 45 & 0.647 & 0.112 & 0.226 & 0.221 \\
\hline Pregunta 46 & 0.611 & 0.233 & 0.174 & 0.110 \\
\hline Pregunta 47 & 0.503 & 0.277 & 0.163 & -0.115 \\
\hline Pregunta 48 & 0.569 & 0.221 & 0.081 & 0.096 \\
\hline Pregunta 49 & 0.535 & 0.090 & 0.264 & -0.104 \\
\hline Pregunta 52 * & 0.345 & 0.499 & -0.151 & 0.105 \\
\hline
\end{tabular}

Nota: Elaboración propia.

Etapa 5: Construcción del instrumento final. A partir del resultado anterior, se construyó el instrumento final compuesto por 26 preguntas, el cual se muestra en la Tabla 8. 


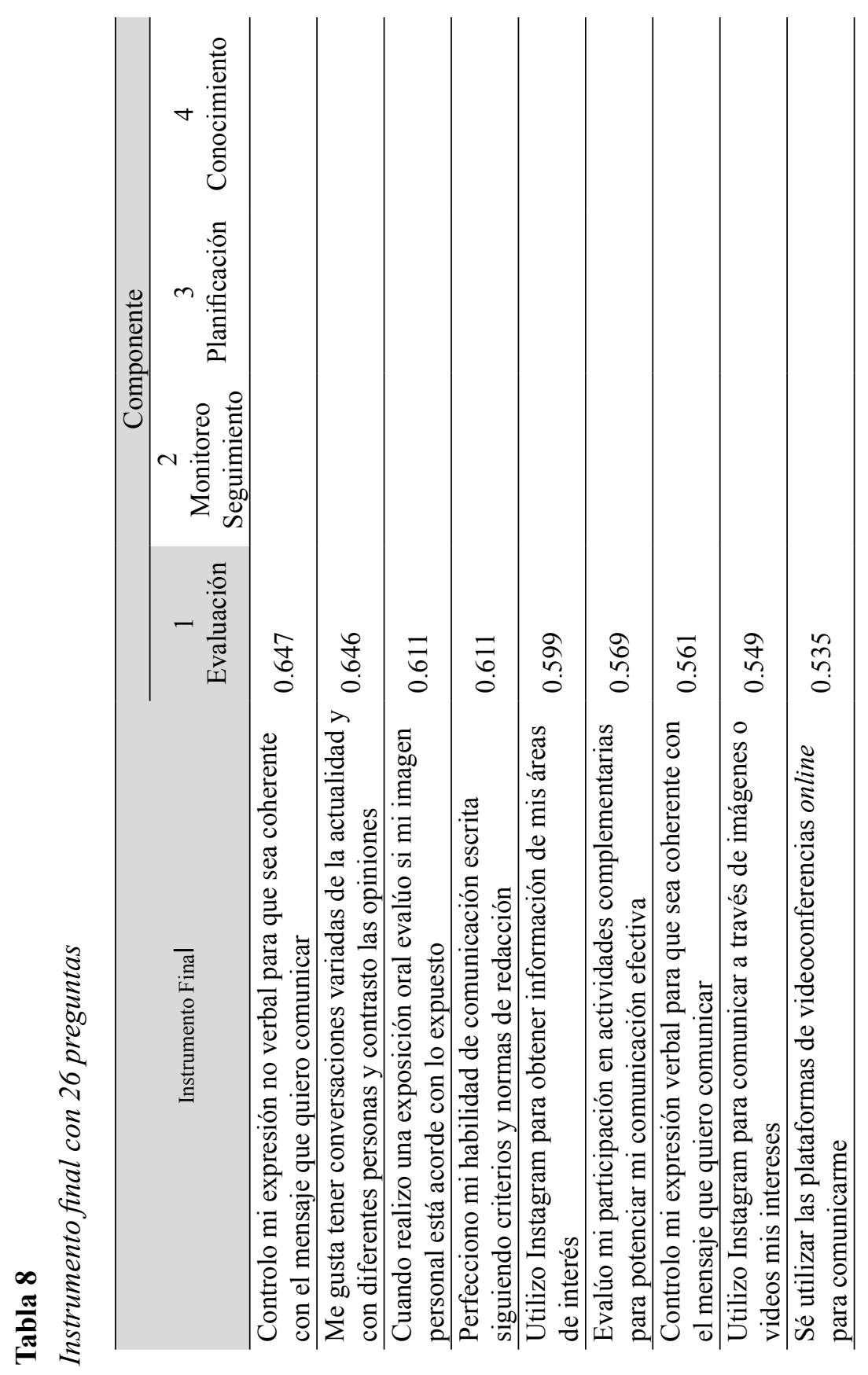




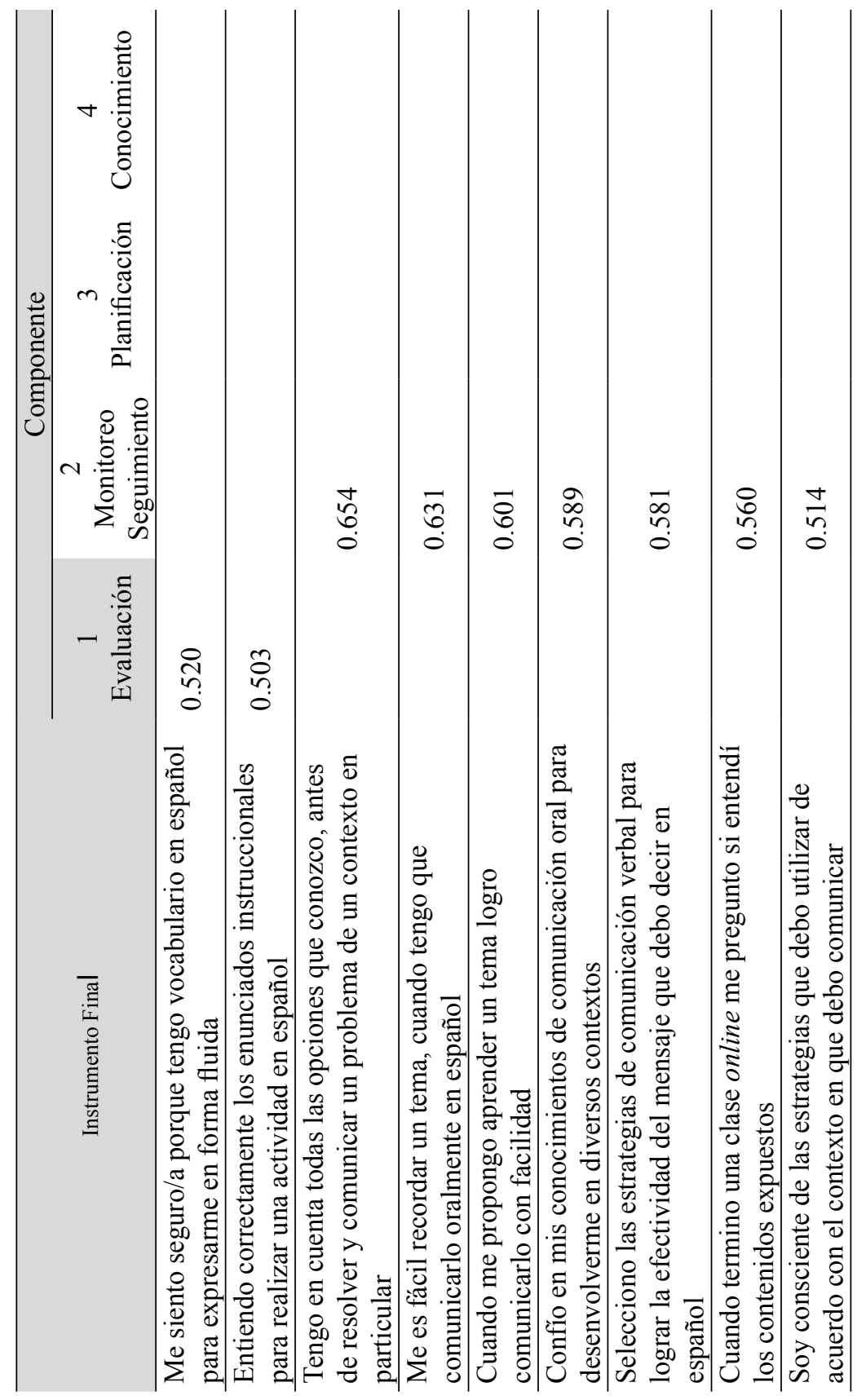




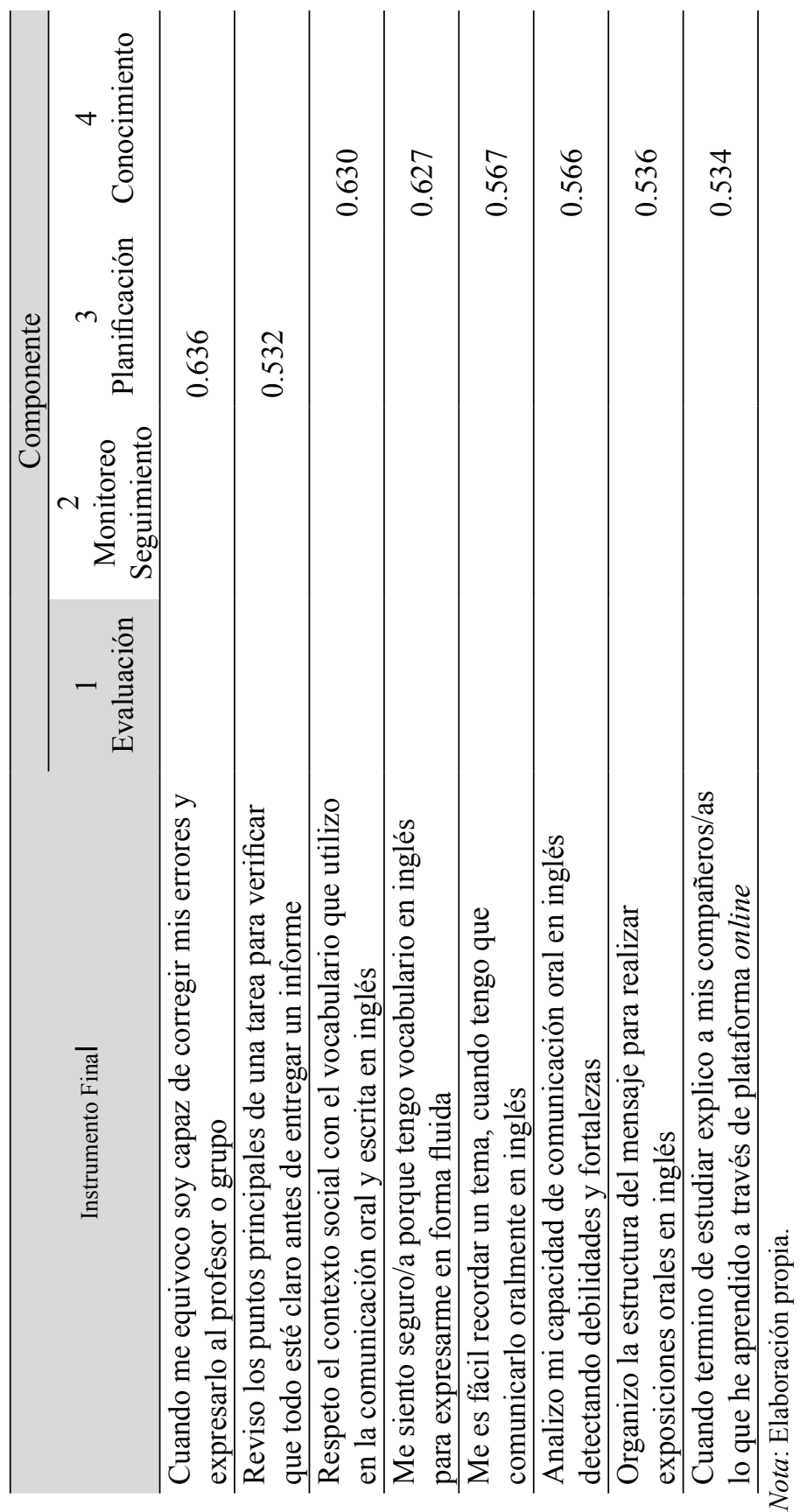


El instrumento final mostrado en la Tabla 8, incluye la matriz de cargas factoriales con componentes rotados y las preguntas seleccionadas agrupadas según su dimensión y ordenadas de mayor a menor en cada dimensión en cuanto a su carga factorial. Se puede visualizar en esta tabla, que el componente 1 se identifica con la dimensión conocimiento, la más importante en el análisis factorial, el cual explica la variabilidad total. El componente 2 se relaciona con la dimensión monitoreo-seguimiento. Los componentes 3 y 4 se podrían relacionar parcialmente con las dimensiones planificación y conocimiento, sin embargo, son más difíciles de asociar a las dimensiones de la encuesta, ya que en estos ítems se mezclan preguntas correspondientes a distintas dimensiones de la encuesta original.

El alfa de Cronbach para el constructo final compuesto de 26 preguntas, se muestra en la Tabla 9. Si bien su valor disminuyó a 0.876 , sigue siendo un parámetro numérico muy alto para ciertamente poder validar la fiabilidad del instrumento.

\section{Tabla 9}

Fiabilidad para 26 las preguntas

\begin{tabular}{|c|c|}
\hline \multicolumn{2}{|c|}{ Estadísticas de fiabilidad } \\
\hline Alfa de Cronbach & N de elementos \\
\hline 0.876 & 26 \\
\hline
\end{tabular}

Nota: Elaboración propia.

Las pruebas KMO y esfericidad de Bartlett son mostradas en la Tabla 10.

Tabla 10

Fiabilidad para 26 las preguntas

\begin{tabular}{|l|c|c|}
\hline \multicolumn{3}{|c|}{ Prueba de KMO y Bartlett } \\
\hline \multicolumn{2}{|c|}{ Medida Kaiser-Meyer-Olkin de adecuación de muestreo } & 0.787 \\
\hline \multirow{2}{*}{$\begin{array}{l}\text { Prueba de esfericidad de } \\
\text { Bartlett }\end{array}$} & $\sim x^{2}$ & 908.675 \\
\cline { 2 - 3 } & $\mathrm{gl}$ & 325 \\
\cline { 2 - 3 } & Sig. & 0.000 \\
\hline
\end{tabular}

Nota: Elaboración propia. 
Podemos ver, de acuerdo con lo expuesto en la Tabla 10, que el valor KMO de adecuación de muestreo subió de 0.722 a 0.787 debido, principalmente, a la eliminación de preguntas con baja ponderación. La significancia en la prueba de esfericidad es altamente adecuada. Al existir coherencia entre ambos parámetros el análisis factorial es adecuado para explicar los datos.

En la Tabla 11, se muestra un extracto de la varianza total explicada por los 4 componentes y las 26 preguntas finales.

De acuerdo con lo mostrado en la Tabla 11, se puede observar que el constructo final mejoró la varianza total explicada con respecto al análisis factorial con 46 preguntas. Las 26 preguntas elegidas explican un $47.19 \%$ de la varianza total contra el $39.21 \%$ logrado anteriormente. El gráfico de sedimentación para este análisis factorial se muestra en la Figura 2, el cual es claramente mejor que el presentado en la Figura 1.

\section{Figura 2}

Gráfico de sedimentación para las 26 preguntas

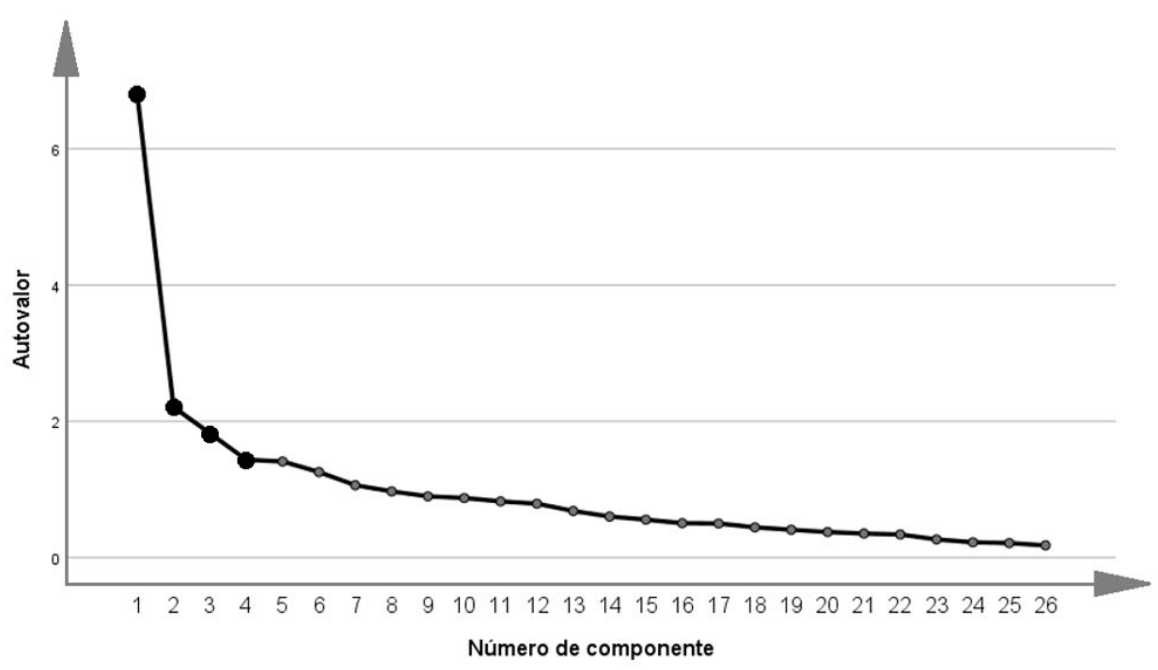

Nota: Elaboración propia.

Finalmente, se construye un instrumento, cuyo Alfa de Cronbach es 0.876 , un valor bastante alto, compuesto de 26 preguntas las cuales explican el $47 \%$ de la varianza. 


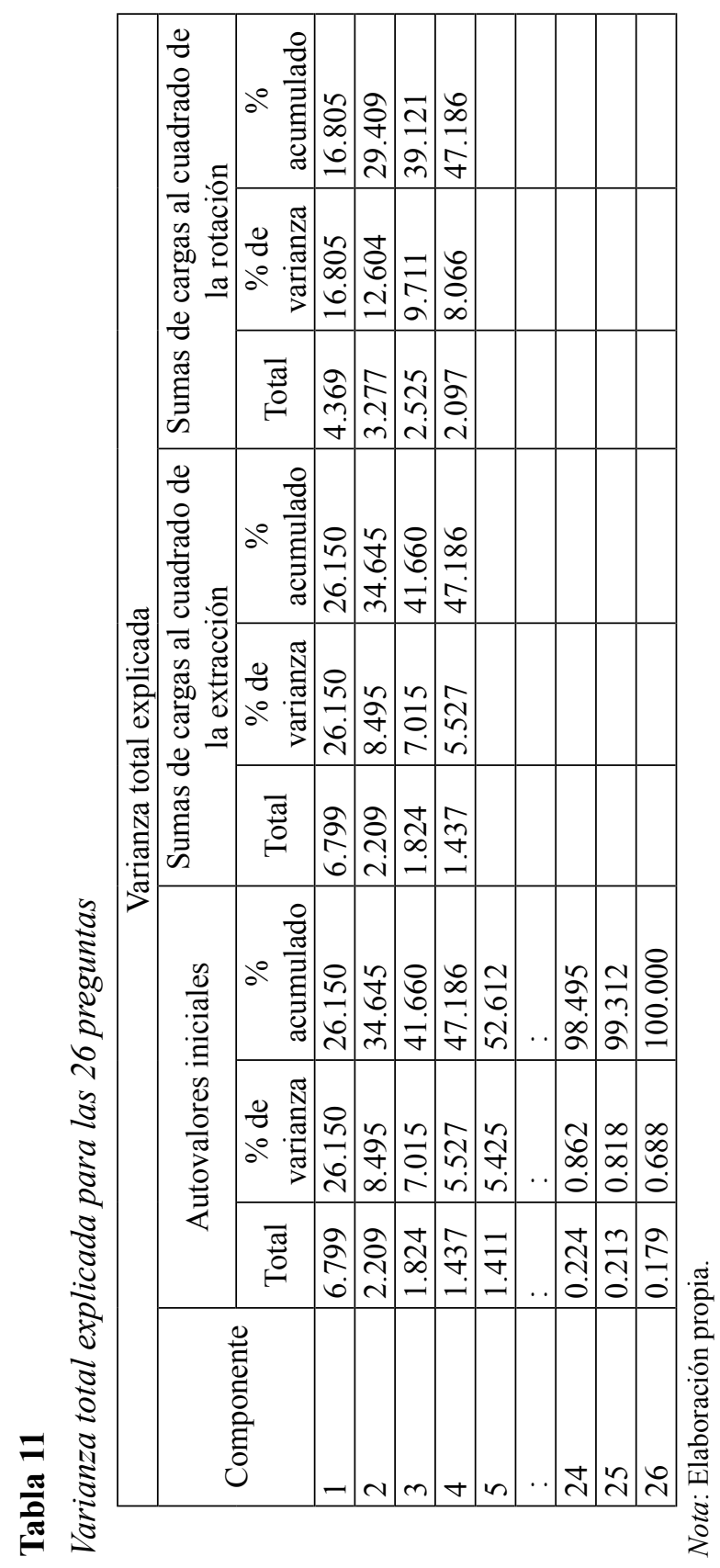




\section{Discusión}

Los conceptos que retrata la bibliografía en aspectos de metacognición son amplísimos, pero no se advierte un consenso en sus definiciones, no obstante, parece relacionarse fuertemente con el proceso de autogestión del conocimiento.

$\mathrm{Al}$ indagar en estudios referentes a experiencias en Chile y Latinoamérica en el desarrollo de instrumentos con la medición de la competencia comunicación a través de la metacognición, estos son escasos, y algunos se refieren principalmente a aspectos muy particulares de la comunicación. Todo indica que la metacognición se puede enseñar y contribuye positivamente a la adquisición de competencias.

Así, es deseable que el estudiantado comprenda que el uso de estrategias metacognitivas contribuye ciertamente al logro de las competencias declaradas en los perfiles de egreso de las distintas carreras universitarias y, naturalmente, a la autogestión del conocimiento.

En esa línea, en este trabajo se planteó una primera aproximación al diseño y validación de un instrumento que permita medir algunos aspectos que debiera desarrollar el estudiantado universitario en cuanto a la competencia comunicativa desde la mirada metacognitiva.

Este instrumento puede aplicarse al interior del aula, debiendo ser complementado con una intervención que apoye la consecución del logro de las competencias en el área comunicación y, por cierto, de una rúbrica que dé cuenta del avance en aquellos aspectos.

El instrumento se compone de un cuestionario que consta de 26 preguntas, agrupadas en 4 dimensiones definidas como conocimiento, planificación, monitoreo/seguimiento y evaluación, explicando en conjunto cerca del $47 \%$ de la varianza global, en el cual el conocimiento más varianza se explica en comparación con las otras tres. El parámetro alfa de Cronbach es adecuado para el instrumento, al presentar un valor numérico de 0.876 . La prueba KMO de adecuación de muestreo presenta un valor numérico de 0.87 , también adecuado para el instrumento. Es importante destacar que los parámetros antes mencionados son un poco menos afortunados que los encontrados por Jaramillo y Osses (2012).

Al aplicar el instrumento a un grupo de estudiantes, se propone el siguiente procedimiento, mostrado en la Figura 3, con el propósito de obtener tres categorías del desarrollo de la competencia comunicativa a 
través de una estrategia metacognitiva, a saber, bajo, medio o alto, tanto total o individual y por cada dimensión propuesta.

\section{Figura 3}

Procedimiento para obtener categorías en el desarrollo de la competencia comunicación

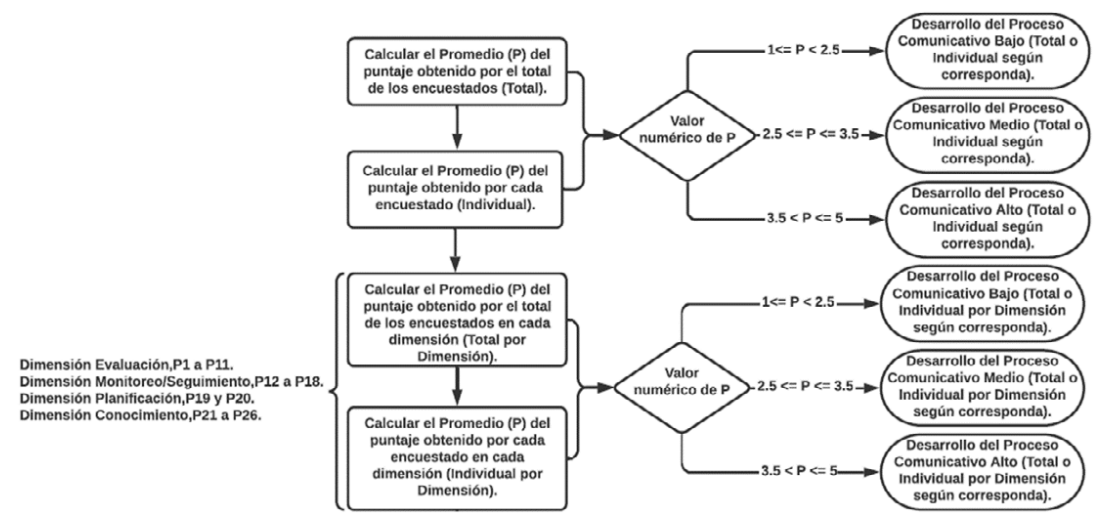

Nota: Desarrollo del proceso.

Si bien con este estudio se ha logrado un avance al diseñar un instrumento, queda pendiente, por un lado, que pueda ser aplicado a una población mayor con el fin de contrastar los resultados obtenidos con los aquí presentados, al recalcular los indicadores estadísticos con el fin de confirmar los resultados y, por otro lado, contrastar la correlación entre este instrumento y otro que mida específicamente la comunicación o la metacognición.

En la Figura 4, se muestra el instrumento de medición de la competencia comunicación a través de estrategias metacognitivas en estudiantes universitarios.

Finalmente, hay que indicar que este trabajo fue desarrollado como parte del Proyecto OEA 19105 de la Universidad Técnica Federico Santa María - Chile. 
Instrumento de medición de la competencia de comunicación a través de estrategias metacognitivas en estudiantado universitario

\section{Figura 4}

\section{Instrumento de medición de la competencia comunicación a través de estrategias metacognitivas en estudiantado universitario}

\section{ENCUESTA SOBRE COMUNICACIÓN UTILIZANDO ESTRATEGIAS METACOGNITIVAS}

Público Objetivo de Aplicación: Estudiantes Universitarios de Pregrado.

Instrucciones para responder la Encuesta:

Estimada y estimado encuestado. La siguiente Encuesta tiene por finalidad conocer las actitudes que usted presenta frente al desarrollo de la Comunicación Utilizando Estrategias Metacognitivas. Así, le solicitamos contestar el siguiente cuestionario marcando en las casillas destinadas a cada aseveración de acuerdo con su preferencia. La puntuación, que va desde 1 hasta 5, se basa en el siguiente criterio:

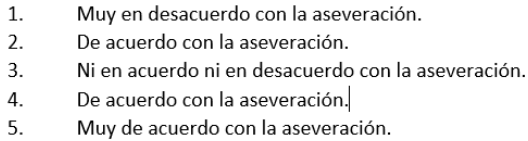

Por ejemplo, si usted está "De acuerdo" con una aseveración, deberá marcar la casilla correspondiente a la columna 4 de esa pregunta.

Responder la encuesta no le tomará más allá de 30 minutos.

\begin{tabular}{|c|c|c|c|c|}
\hline & Aseveración & \begin{tabular}{|l|l|l|l}
1 & 2 \\
\end{tabular} & 23 & 4 \\
\hline P1 & Controlo mi expresión no verbal para que sea coherente con el mensaje que quiero comunicar. & & & \\
\hline P2 & $\begin{array}{l}\text { Me gusta tener conversaciones variadas de la actualidad y con diferentes personas y contrasto } \\
\text { las opiniones. }\end{array}$ & & & \\
\hline P3 & Cuando realizo una exposición oral evalúo si mi imagen personal está acorde con lo expuesto. & & & \\
\hline P4 & Perfecciono mi habilidad de comunicación escrita siguiendo criterios y normas de redacción. & & & \\
\hline P5 & Utilizo Instagram para obtener información de mis áreas de interés. & & & \\
\hline P6 & $\begin{array}{l}\text { Evalúo mi participación en actividades complementarias para potenciar mi comunicación } \\
\text { efectiva. }\end{array}$ & & & \\
\hline P7 & Controlo mi expresión verbal para que sea coherente con el mensaje que quiero comunicar. & & & \\
\hline P8 & Utilizo Instagram para comunicar a través de imágenes o videos mis intereses. & & & \\
\hline P9 & Sé utilizar las plataformas de videoconferencias on line para comunicarme. & & & \\
\hline P10 & Me siento segura(o) porque tengo vocabulario en español para expresarme en forma fluida. & & & \\
\hline P11 & Entiendo correctamente los enunciados instruccionales para realizar una actividad en español. & & & \\
\hline P12 & $\begin{array}{l}\text { Tengo en cuenta todas las opciones que conozco, antes de resolver y comunicar un problema } \\
\text { de un contexto en particular. }\end{array}$ & & & \\
\hline P13 & Me es fácil recordar un tema, cuando tengo que comunicarlo oralmente en español. & & & \\
\hline P14 & Cuando me propongo aprender un tema logro comunicarlo con facilidad. & & & \\
\hline P15 & Confío en mis conocimientos de comunicación oral para desenvolverme en diversos contextos. & & & \\
\hline P16 & $\begin{array}{l}\text { Selecciono las estrategias de comunicación verbal para lograr la efectividad del mensaje que } \\
\text { debo decir en español. }\end{array}$ & & & \\
\hline P17 & Cuando termino una clase on line me pregunto si entendí los contenidos expuestos. & & & \\
\hline P18 & $\begin{array}{l}\text { Soy consciente de las estrategias que debo utilizar de acuerdo con el contexto en que debo } \\
\text { comunicar. }\end{array}$ & & & \\
\hline P19 & Cuando me equivoco soy capaz de corregir mis errores y expresarlo al profesor o grupo. & & & \\
\hline P20 & $\begin{array}{l}\text { Reviso los puntos principales de una tarea para verificar que todo esté claro antes de entregar } \\
\text { un informe. }\end{array}$ & & & \\
\hline P21 & $\begin{array}{l}\text { Respeto el contexto social con el vocabulario que utilizo en la comunicación oral y escrita en } \\
\text { inglés. }\end{array}$ & & & \\
\hline P22 & Me siento segura (o) porque tengo vocabulario en inglés para expresarme en forma fluida. & & & \\
\hline P23 & Me es fácil recordar un tema, cuando tengo que comunicarlo oralmente en inglés. & & & \\
\hline P24 & Analizo mi capacidad de comunicación oral en inglés detectando debilidades y fortalezas. & & & \\
\hline P25 & Organizo la estructura del mensaje para realizar exposiciones orales en inglés. & & & \\
\hline P26 & $\begin{array}{l}\text { Cuando termino de estudiar explico a mis compañeros lo que he aprendido a través de } \\
\text { plataforma online. }\end{array}$ & & & \\
\hline
\end{tabular}

Nota: Elaboración propia. 


\section{Referencias}

Arráez, L., Nuñez J., Carabantes D., Lozano R., Iglesias I., Palacios E., Del Castillo B. y Nogales A. (2008). Adquisición de competencias transversales en alumnos de pregrado de ciencias de la salud en la Universidad Complutense: Una experiencia positiva. Revista de Educación Médica, 11(3), 169-177. http://scielo.isciii. es/pdf/edu/v11n3/original3.pdf

Bonilla, M. y Díaz, C. (2018). La metacognición en el aprendizaje de una segunda lengua: Estrategias, instrumentos y evaluación. Revista Educación, 42(2), 4-8. http://www.redalyc.org/articulo. oa? $\mathrm{id}=44055139018$

Canales, T. y Schmal, R. (2013). Trabajando con pósteres: Una herramienta para el desarrollo de habilidades de comunicación en la educación de pregrado. Formación Universitaria, 6(1), 41-52. http://dx.doi.org/10.4067/S0718-50062013000100006

De la Uz, M., Lemus A., Valdés M. y Padrón, C. (2010). Competencia comunicativa en los estudiantes de medicina: Diagnostico preliminar. Revista Ciencias Médicas, 14(1), 43-49. http://scielo.sld. cu/scielo.php?pid=S1561-31942010000100031\&script=sci_arttext\&tlng=en

Di Pardo, D. (2019). Going Beyond Words and Actions: Teaching Metacognitive and Soft Skills to ESP Communication Students at the Dawn of the Fourth Industrial Revolution. En S. Papadima-Sophocleous, E. Kakoulli y C. N. Giannikas (Eds.), ESP TEACHING AND TEACHER EDUCATION: CURRENT THEORIES AND PRACTICES (pp. 147-161). Research-Publishing.net. https://doi.org/10.14705/rpnet.2019.33.931

Escobari, M. (2013). Nuevas herramientas para los alumnos de $5^{\circ}$ año de la carrera de Psicología. Revista De Psicología, 9, 27-46. http://www.scielo.org.bo/scielo.php?pi$\mathrm{d}=\mathrm{S} 2223-30322013000100004 \&$ script $=$ sci_abstract\&tlng=pt

Flavell, J. (1976). Metacognitive aspects of problem solving. The nature of intelligence. Hillsdale. Erlbaum. RESNICK, LAUREN 230-240.

Godoy, O. (2016). Análisis de competencias y problemas frecuentes en la comunicación oral en estudiantes de octavo año de enseñanza básica [Tesis de maestría no publicada]. Universidad de Chile. 
http://repositorio.uchile.cl/bitstream/handle/2250/140615/Analisis-de-competencias-y-problemas-frecuentes-en-la-comunicaci\% $\mathrm{C} 3 \% \mathrm{~B} 3 n$-oral-en-estudiantes-de-octavo-ano-de-ensenanza-basica.pdf?sequence $=1$

González, L., Álvarez, M. y Reyna, M. (2015). Análisis epistemológico de la comunicación oral del inglés con fines profesionales. Medisan, 19(3), 444-450. http://scielo.sld.cu/scielo.php?pi$\mathrm{d}=\mathrm{S} 1029-30192015000300019 \&$ script $=\mathrm{sci}$-arttext\&tlng $=\mathrm{pt}$

González, R., Reynoso, M., Soto, F., Méndez, J., Alonso, N., Felix, C. y López M. (2017). Habilidades metacognitivas en alumnos del Curso de Especialización en Medicina Familiar. Revista FEM, 20(4), 177-181. http://scielo.isciii.es/scielo. php?script=sci_arttext\&pid=S2014-98322017000400004

Huertas, A., Bravo, V., Judith, G. y Galindo, M. (2014). Validación del instrumento: Inventario de habilidades metacognitivas (MAI) con estudiantes colombianos. Praxis \& Saber, 5(10), 55-74. http://www.scielo.org.co/scielo.php?pi$\mathrm{d}=\mathrm{s} 2216-01592014000200004 \&$ script=sci_abstract\&tlng=es

Jaramillo, S. y Osses, S. (2012). Validación de un Instrumento sobre Metacognición para Estudiantes de Segundo Ciclo de Educación General Básica. Estudios Pedagógicos, 38(2),117-131. https://scielo.conicyt.cl/scielo. php?script=sci_arttext\&pid=S0718-07052012000200008

Jurado, M., Bravo, G. y Avello, R. (2017). Propuesta de un sistema de métodos para la comunicación interpersonal en el contexto de la educación superior. Medisur, 15(6), 860-869. https://www.medigraphic.com/cgi-bin/new/resumen.cgi?IDARTICULO=78729

Liyanage, I., Bartlett, B., Birch, G. y Tao, T. (2012). "To be or not to be" metacognitive: learning EFL strategically, Electronic journal of foreign language teaching, 9(1), 5-25. http://dro.deakin.edu.au/ eserv/DU:30070974/liyanage-tobeornot-2012.pdf

Marín, N. (2017). Comunicación interpersonal en los equipos de mentores de la Facultad de Ciencias de la Educación y de la Facultad de Enfermería, Fisioterapia y Podología [Tesis de maestría no publicada]. Universidad de Sevilla. https://idus.us.es/bitstream/ handle/11441/70747/TFM\%20MAR\%c3\%8dN\%20DOM\%c3\%8dNGUEZ\%2c\%20NURIA.pdf?sequence $=1 \&$ isAllowed $=\mathrm{y}$ 
Mokhtari, K. y Reichard, C. A. (2002). Evaluar la conciencia metacognitiva de los estudiantes sobre las estrategias de lectura. Revista de Psicología Educativa, 94(2), 249-259. https://doi. org/10.1037/0022-0663.94.2.249

Osses, S., Salamé, M., y Gálvez, J. (2010). Hacia una educación de calidad en el ámbito científico. Autonomía en el aprendizaje a través de la metacognición. Ponencia presentada en el Congreso Iberoamericano de Educación Metas 2021. Buenos Aires, Argentina. https://www.adeepra.org.ar/congresos/Congreso\%20IBEROAMERICANO/ACCESO/RLE2610_Osses.pdf

Palacios, A. y Schinella, G. (2017). Diseño y validación de un instrumento para evaluar la metacognición sobre el estudio en estudiantes de medicina. Revista de Educación de La Universidad De Granada, 24, 9-28. http://reugra.es/index.php/reugra/article/ view/70

Rico, A. y Níkleva, D. (2016). Análisis de la competencia lingüístico-discursiva escrita de los alumnos de nuevo ingreso del grado de maestro en educación primaria. Revista Signos. Estudios De Lingüistica, 49(90), 48-70. doi.org/10.4067/S0718-09342016000100003

Ramírez, J. y Pereira, S. (2006). Adaptación de un instrumento para evaluar el conocimiento de estrategias metacognitivas de estudiantes universitarios venezolanos al leer textos académicos en inglés. Laurus, 12, 148-169. http://www.redalyc.org/articulo. oa? $\mathrm{id}=76109909$

Rincón, J. (2011). La importancia de la comunicación no verbal en la enseñanza. Revista Ingeniería Solidaria, 6(10), 113-120. https:// revistas.ucc.edu.co/index.php/in/article/download/460/465/

Roca, F., Serrano L. y Cuba O. (2005). Un modelo interactivo para la comunicación sociopsicológica. Humanidades médicas, 5(2), 2-12. http://scielo.sld.cu/scielo.php?script=sci_arttext\&pi$\mathrm{d}=\mathrm{S} 1727-81202005000200005 \& \operatorname{lng}=$ es\&tlng=es

Ruiz, E., Cruz J. y Méndez V. (2018). Análisis secuencial del discurso: Conducta no verbal y su relevancia discursiva durante la toma de turnos en clases de segunda lengua. Perfiles Educativos, 40(160), 141-154. http://www.scielo.org.mx/scielo.php?script=sci_abstract\&pid=S0185-26982018000200141\&lng=es\&nrm=iso\&tlng $=$ es 
Schraw, G. y Moshman, D. (1995). Metacognitive Theories. Educational Psychology Review, 7(4), 351-371. https://digitalcommons.unl. edu/cgi/viewcontent.cgi?article=1040\&context=edpsychpapers

Sáiz, M. y Guijo, V. (2010). Competencias y estrategias metacognitivas en educación infantil: Un camino hacia el desarrollo de procedimientos de resolución de problemas. Revista de Psicología, 2, 497-504. http://dehesa.unex.es/handle/10662/3125

Tejera, J. y Cardoso, M. (2005). Tratamiento de la habilidades comunicativas en el contexto universitario. Revista Universal y Sociedad, 7(3), 168-172. http://scielo.sld.cu/scielo. php?script=sci_arttext\&pid=S2218-36202015000200024

Tesouro, M. (2005). La metacognición en la escuela: La importancia de enseñar a pensar. Educar, 35, 135-144. https://www.redalyc.org/ pdf/3421/342130824013.pdf

Tovar, J. (2008a). Un modelo metacognitivo como integrador de: competencias, aprendizaje por investigación y relaciones entre ciencia, tecnología, sociedad y ambiente -CTSA-, en la enseñanza de la química. V Simposio Universitario Iberoamericano sobre Medio Ambiente (SUIMA 2008). Enseñanza de la Química. https:// doi.org/10.13140/2.1.2579.6161

Tovar, J. (2008b). Modelo metacognitivo como integrador de estrategias de enseñanza y estrategias de aprendizaje de las ciencias y su relación con las competencias. Revista Iberoamericana de Educación, 46(7), 1-9. https://www.researchgate.net/profile/ Julio_Tovar-Galvez/publication/28230589_Modelo_metacognitivo_como_integrador_de_estrategias_de_ensenanza_y_estrategias_de_aprendizaje_de_las_ciencias_y_su_relacion_con las_competencias/links/0a85e5324e9dd9f0d7000000.pdf

Troitiño, D. y Hernández, A. (2015). Propuesta de actividades para el desarrollo de la expresión oral en inglés en la Escuela de Energía y Minas. Revista Cubana De Educación Superior, 34(3), 4-12. http://scielo.sld.cu/scielo. php?script=sci_arttext\&pid=S0257-43142015000300001

Verano-Tacoronte, D., González -Betancor, S., Bolívar-Cruz, A., Fernández-Monroy, M. y Galván-Sánchez, I. (2016). Valoración de la competencia de comunicación oral de estudiantes universitarios a través de una rúbrica fiable y válida. Revista 
Brasileira de Educação, 21(64), 39-60. https://doi.org/10.1590/ S1413-24782016216403

Yilorm, Y. (2016). Proceso de enseñanza aprendizaje de la lengua inglesa en escuelas públicas chilenas: ¿Producción o reproducción? Estudios Pedagógicos, 42, 104-110. https://scielo.conicyt.cl/ scielo.php?script $=$ sci arttext\&pid=S0718-07052016000300009

Zanaton, H., Zakaria, E., Mohd, T., Osman, K., Choon, D., Diyana, S. y Krish, P. (2012). Comunication Skills Among University Students. Procedia - Social and Behavioral Sciences, 59, 71-76. https://doi.org/10.1016/j.sbspro.2012.09.247 